(C) by R. Oldenbourg Verlag, München 1997

\title{
Crystal structure of dihydroergocristine bis(dioxane) solvate, $\left(\mathrm{C}_{35} \mathrm{H}_{41} \mathrm{~N}_{5} \mathrm{O}_{5}\right)\left(\mathrm{C}_{4} \mathrm{H}_{8} \mathrm{O}_{2}\right)_{2}$
}

\section{J. Cejka, B. Kratochvíl}

Prague Institute of Chemical Technology, Department of Solid State Chemistry, Technicka 5, 16628 Prague 6, Czech Republic

A. Jegorov

Galena Co., Research Unit, Branišovska 31, 37005 Ceské Budejovice, Czech Republic

and L. Cvak

Galena Co., R. \& D., 74770 Opava Komarov, Czech Republic

Received June 20, 1996, CSD-No. 402529

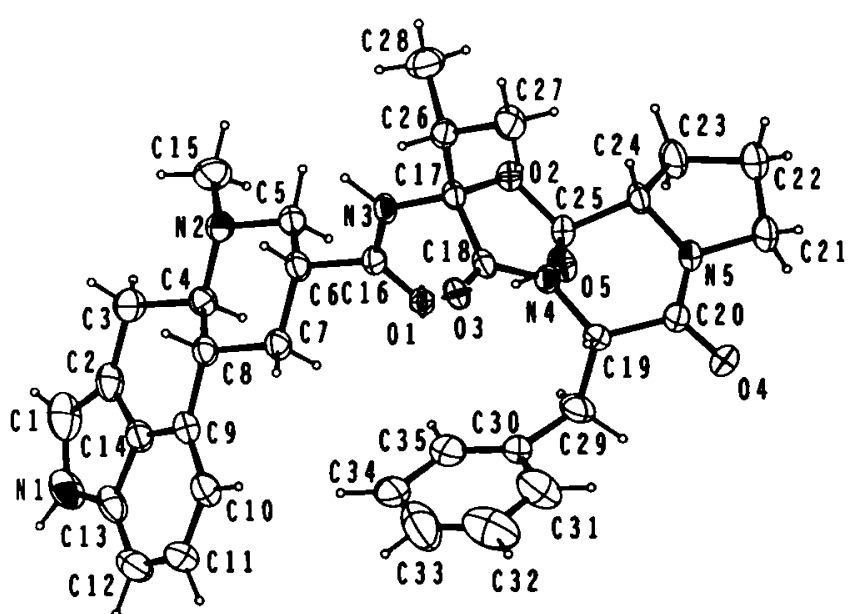

Source of material: Dihydroergocristine (Galena Co., Czech Republic) was dissolved in dioxane at $353 \mathrm{~K}(140 \mathrm{mg} / 2 \mathrm{ml})$ and the solution was allowed to cool. After 24 hours crystals were separated, washed with dioxane and dried in air.

The title compound crystallizes as a solvate with two slightly disordered dioxane molecules.

$\mathrm{C}_{43} \mathrm{H}_{57} \mathrm{~N}_{5} \mathrm{O}$, monoclinic, $P 12{ }_{1} 1$ (No. 4), $a=10.0503(9) \AA$, $b=10.553(1) \AA, c=19.743(2) \AA, \beta=92.954(7)^{\circ}, V=2091.2 \AA^{3}$, $Z=2, R(F)=0.073, R_{\mathrm{w}}(F)=0.087$.

Table 1. Parameters used for the $\mathrm{X}$-ray data collection

\begin{tabular}{ll}
\hline Crystal: & colorless prims, size $0.43 \times 0.56 \times 0.69 \mathrm{~mm}$ \\
Wavelength: & Cu $K_{\alpha}$ radiation $(1.54183 \AA)$ \\
$\mu:$ & $0.68 \mathrm{~cm}^{-1}$ \\
Diffractometer: & Enraf-Nonius CAD4 \\
Scan mode: & $2 \theta$ \\
$\mathrm{T}_{\text {measurement: }}$ & $293 \mathrm{~K}$ \\
$2 \theta_{\text {max }}:$ & $55^{\circ}$ \\
$\mathrm{N}(h k l)_{\text {unique: }}$ & 2793 \\
Criterion for $F_{\mathrm{o}}:$ & $F_{\mathrm{o}}>1.96 \sigma\left(F_{0}\right)$ \\
N(param $)_{\text {refined: }}$ & 513 \\
Program: & CRYSTALS \\
\hline
\end{tabular}

Table 2. Final atomic coordinates and displacement parameters (in $\AA^{2}$ )

\begin{tabular}{|c|c|c|c|c|c|}
\hline Atom & Site & $x$ & $y$ & $z$ & $U_{\text {iso }}$ \\
\hline$H(11)$ & $2 a$ & $1.2385(6)$ & $0.8365(8)$ & $0.4929(3)$ & 0.08446 \\
\hline H(31) & $2 a$ & $1.1618(4)$ & $1.0892(5)$ & $0.4408(2)$ & 0.0618 \\
\hline$H(32)$ & $2 a$ & $1.2019(4)$ & $1.0421(5)$ & $0.3658(2)$ & 0.06188 \\
\hline$H(41)$ & $2 a$ & $0.9393(4)$ & $1.1179(4)$ & $0.4029(2)$ & 0.04779 \\
\hline H(51) & $2 a$ & $0.9406(4)$ & $1.3874(4)$ & $0.3011(2)$ & 0.05363 \\
\hline$H(52)$ & $2 a$ & $0.8542(4)$ & $1.3071(4)$ & $0.3542(2)$ & 0.05363 \\
\hline$H(61)$ & $2 a$ & $0.9044(3)$ & $1.2282(4)$ & $0.2184(2)$ & 0.04538 \\
\hline$H(71)$ & $2 a$ & $0.7451(4)$ & $1.0976(4)$ & $0.3144(2)$ & 0.0518 \\
\hline$H(72)$ & $2 a$ & $0.7766(4)$ & $1.0447(4)$ & $0.2391(2)$ & 0.0518 \\
\hline$H(81)$ & $2 a$ & $1.0039(3)$ & $1.0162(4)$ & $0.2746(2)$ & 0.04378 \\
\hline$H(101)$ & $2 a$ & $0.7453(5)$ & $0.8409(5)$ & $0.2711(2)$ & 0.05891 \\
\hline H(111) & $2 a$ & $0.7210(5)$ & $0.6346(5)$ & $0.3164(3)$ & 0.06860 \\
\hline H(121) & $2 a$ & $0.8725(5)$ & $0.5531(5)$ & $0.4035(3)$ & 0.06962 \\
\hline$H(151)$ & $2 a$ & $1.1301(5)$ & $1.3964(6)$ & $0.3669(3)$ & 0.0738 \\
\hline$H(152)$ & $2 a$ & $1.1992(5)$ & $1.2707(6)$ & $0.4019(3)$ & 0.0738 \\
\hline$H(153)$ & $2 a$ & $1.0617(5)$ & $1.3273(6)$ & $0.4305(3)$ & 0.0738 \\
\hline H(191) & $2 a$ & $0.2575(4)$ & $1.3368(4)$ & $0.1118(2)$ & 0.05098 \\
\hline$H(211)$ & $2 a$ & $0.0511(5)$ & $1.6979(5)$ & $0.1914(3)$ & 0.07379 \\
\hline$H(212)$ & $2 a$ & $0.0601(5)$ & $1.6355(5)$ & $0.2671(3)$ & 0.07379 \\
\hline$H(221)$ & $2 a$ & $0.1721(5)$ & $1.8135(6)$ & $0.2954(3)$ & 0.07863 \\
\hline$H(222)$ & $2 a$ & $0.2113(5)$ & $1.8397(6)$ & $0.2174(3)$ & 0.07863 \\
\hline$H(231)$ & $2 a$ & $0.4164(5)$ & $1.7726(5)$ & $0.2656(3)$ & 0.07140 \\
\hline$H(232)$ & $2 a$ & $0.3453(5)$ & $1.6672(5)$ & $0.3124(3)$ & $0.0714 \mathrm{C}$ \\
\hline$H(241)$ & $2 a$ & $0.3739(4)$ & $1.6566(4)$ & $0.1689(2)$ & 0.04608 \\
\hline$H(261)$ & $2 a$ & $0.7197(4)$ & $1.4825(4)$ & $0.0665(2)$ & 0.04813 \\
\hline $\mathbf{H}(271)$ & $2 a$ & $0.6019(5)$ & $1.6593(5)$ & $0.0215(2)$ & 0.06444 \\
\hline$H(272)$ & $2 a$ & $0.5145(5)$ & $1.6645(5)$ & $0.0889(2)$ & 0.06444 \\
\hline H(273) & $2 a$ & $0.5016(5)$ & $1.5428(5)$ & $0.0380(2)$ & 0.0644 \\
\hline$H(281)$ & $2 a$ & $0.8111(5)$ & $1.6867(6)$ & $0.0865(3)$ & 0.0834 \\
\hline$H(282)$ & $2 a$ & $0.7364(5)$ & $1.6930(6)$ & $0.1579(3)$ & 0.08349 \\
\hline$H(283)$ & $2 a$ & $0.8542(5)$ & $1.5891(6)$ & $0.1479(3)$ & 0.08349 \\
\hline$H(291)$ & $2 a$ & $0.3019(6)$ & $1.2513(5)$ & $0.2472(3)$ & 0.08023 \\
\hline$H(292)$ & $2 a$ & $0.1511(6)$ & $1.2302(5)$ & $0.2144(3)$ & 0.08023 \\
\hline$H(311)$ & $2 a$ & $0.1200(9)$ & $1.1180(7)$ & $0.1096(4)$ & $0.1000 ?$ \\
\hline H(321) & $2 a$ & $0.170(1)$ & $0.937(1)$ & $0.0438(5)$ & 0.16871 \\
\hline H(341) & $2 a$ & $0.5341(9)$ & $0.9043(7)$ & $0.1546(7)$ & 0.12592 \\
\hline$H(351)$ & $2 a$ & $0.4741(7)$ & $1.0926(7)$ & $0.2249(7)$ & 0.11900 \\
\hline H(521) & $2 a$ & $0.8796(8)$ & $1.1958(9)$ & $-0.0638(4)$ & 0.1099 \\
\hline H(522) & $2 a$ & $0.9676(8)$ & $1.0917(9)$ & $-0.0198(4)$ & 0.10996 \\
\hline H(531) & $2 a$ & $0.935(1)$ & $1.2699(8)$ & $0.0457(4)$ & 0.11696 \\
\hline$H(532)$ & $2 a$ & $0.775(1)$ & $1.2451(8)$ & $0.0372(4)$ & 0.11696 \\
\hline H(551) & $2 a$ & $0.6786(8)$ & $1.088(1)$ & $0.0778(4)$ & 0.13244 \\
\hline H(552) & $2 a$ & $0.7595(8)$ & $0.977(1)$ & $0.1203(4)$ & 0.13244 \\
\hline$H(561)$ & $2 a$ & $0.858(1)$ & $0.916(1)$ & $0.0236(4)$ & 0.13423 \\
\hline$H(562)$ & $2 a$ & $0.696(1)$ & $0.907(1)$ & $0.0129(4)$ & 0.13423 \\
\hline H(621) & $2 a$ & $0.368(1)$ & $0.605(2)$ & $0.4116(5)$ & 0.18075 \\
\hline$H(622)$ & $2 a$ & $0.416(1)$ & $0.467(2)$ & $0.4410(5)$ & 0.18075 \\
\hline$H(631)$ & $2 a$ & $0.499(1)$ & $0.634(3)$ & $0.516(2)$ & 0.28798 \\
\hline H(632) & $2 a$ & $0.590(1)$ & $0.615(3)$ & $0.450(2)$ & 0.28798 \\
\hline H(651) & $2 a$ & $0.477(1)$ & $0.523(2)$ & $0.5952(5)$ & 0.17658 \\
\hline$H(652)$ & $2 a$ & $0.506(1)$ & $0.371(2)$ & $0.5991(5)$ & 0.17658 \\
\hline
\end{tabular}


Table 2. (Continued)

\begin{tabular}{llllll}
\hline Atom & Site & $x$ & $y$ & $z$ & $U_{\text {iso }}$ \\
\hline$H(661)$ & $2 a$ & $0.283(1)$ & $0.429(2)$ & $0.5786(6)$ & 0.18235 \\
$H(662)$ & $2 a$ & $0.344(1)$ & $0.358(2)$ & $0.5135(6)$ & 0.18235 \\
$H(331)$ & $2 a$ & $0.376(1)$ & $0.837(1)$ & $0.0661(5)$ & 0.16036 \\
$H(851)$ & $2 a$ & $0.4875(3)$ & $1.3867(3)$ & $0.2851(1)$ & 0.05000 \\
$H(731)$ & $2 a$ & $0.8195(3)$ & $1.3959(3)$ & $0.1715(2)$ & 0.05000 \\
$H(711)$ & $2 a$ & $1.1056(5)$ & $0.6323(5)$ & $0.4848(2)$ & 0.08272 \\
\hline
\end{tabular}

\section{References}

1. Altomare, A.; Cascarano, G.; Giacovazzo G.; Guagliardi A.; Burla M. C. Polidori, G.; Camalli, M.: SIR92 - a program for automatic solution of crystal structures by direct methods. J. Appl. Crystallogr. 27 (1994) $435-436$.

2. Carnthers, J. R.; Watkin, D. J.: A weighting scheme for least-squares structure refinement. Acta Crystallogr. A35 (1979) 698-699.

3. Watkin, D. J.; Prout, C. K.; Carruthers, R. J.; Betteridge, P.: CRYSTALS Issue 10. Chemical Crystallography Laboratory, Oxford, UK 1996.

4. Watkin, D. J.; Prout, C. K.; Pearce, L. J.: CAMERON. Chemical Crystallography Laboratory, Oxford, UK 1996.

5. Watkin, D. J.; Prout, C. K.; Lilley, P. M.deQ.: RC93. Chemical Crystallography Laboratory, Oxford, UK 1994.

Table 3. Final atomic coordinates and displacement parameters (in $\AA^{2}$ )

\begin{tabular}{|c|c|c|c|c|c|c|c|c|c|c|}
\hline Atom & Site & $x$ & $y$ & $z$ & $U_{11}$ & $U_{22}$ & $U_{33}$ & $U_{12}$ & $U_{13}$ & $U_{23}$ \\
\hline $\mathbf{N}(1)$ & $2 a$ & $1.0852(5)$ & $0.7101(5)$ & $0.4573(2)$ & $0.100(3)$ & $0.072(3)$ & $0.078(3)$ & $0.027(3)$ & $0.001(2)$ & $0.028(3)$ \\
\hline $\mathbf{N}(2)$ & $2 a$ & $1.0357(3)$ & $1.2331(4)$ & $0.3394(2)$ & $0.042(2)$ & $0.053(2)$ & $0.061(2)$ & $0.003(2)$ & $-0.007(1)$ & $0.006(2)$ \\
\hline $\mathbf{N}(3)$ & $2 a$ & $0.7314(3)$ & $1.3840(3)$ & $0.1850(2)$ & $0.037(2)$ & $0.047(2)$ & $0.058(2)$ & $0.016(1)$ & $0.005(1)$ & $0.011(2)$ \\
\hline $\mathbf{N}(4)$ & $2 a$ & $0.4003(3)$ & $1.4090(3)$ & $0.1681(1)$ & $0.038(2)$ & $0.044(2)$ & $0.046(2)$ & $0.006(1)$ & $0.003(1)$ & $0.005(1)$ \\
\hline $\mathbf{N}(5)$ & $2 a$ & $0.2048(3)$ & $1.5693(4)$ & $0.2045(2)$ & $0.040(2)$ & $0.047(2)$ & $0.088(2)$ & $0.013(2)$ & $0.016(2)$ & $0.004(2)$ \\
\hline$O(1)$ & $2 a$ & $0.6147(3)$ & $1.2790(3)$ & $0.2623(2)$ & $0.043(1)$ & $0.061(2)$ & $0.085(2)$ & $0.015(1)$ & $0.011(1)$ & $0.023(2)$ \\
\hline$O(2)$ & $2 a$ & $0.5678(2)$ & $1.5397(3)$ & $0.2061(1)$ & $0.043(1)$ & $0.051(2)$ & $0.050(1)$ & $0.008(1)$ & $0.001(1)$ & $-0.003(1)$ \\
\hline$O(3)$ & $2 a$ & $0.5043(3)$ & $1.3020(3)$ & $0.0845(1)$ & $0.067(2)$ & $0.053(2)$ & $0.061(2)$ & $0.002(1)$ & $0.013(1)$ & $-0.012(2)$ \\
\hline$O(4)$ & $2 a$ & $0.0432(3)$ & $1.4320(4)$ & $0.1719(2)$ & $0.041(2)$ & $0.066(2)$ & $0.157(3)$ & $-0.002(2)$ & $0.019(2)$ & $0.007(3)$ \\
\hline$O(5)$ & $2 a$ & $0.4304(3)$ & $1.4561(3)$ & $0.2852(1)$ & $0.069(2)$ & $0.062(2)$ & $0.042(1)$ & $0.021(2)$ & $0.011(1)$ & $0.010(1)$ \\
\hline$C(1)$ & $2 a$ & $1.1575(6)$ & $0.8211(8)$ & $0.4625(3)$ & $0.085(4)$ & $0.120(6)$ & $0.065(3)$ & $0.039(4)$ & $-0.012(3)$ & $0.024(3)$ \\
\hline$C(2)$ & $2 a$ & $1.0988(4)$ & $0.9085(6)$ & $0.4176(2)$ & $0.056(3)$ & $0.076(3)$ & $0.059(2)$ & $0.022(3)$ & $-0.004(2)$ & $0.015(3)$ \\
\hline$C(3)$ & $2 a$ & $1.1303(4)$ & $1.0419(5)$ & $0.3991(2)$ & $0.053(2)$ & $0.066(3)$ & $0.061(2)$ & $0.002(2)$ & $-0.014(2)$ & $0.008(2)$ \\
\hline$C(4)$ & $2 a$ & $1.0050(4)$ & $1.1062(4)$ & $0.3670(2)$ & $0.049(2)$ & $0.047(2)$ & $0.045(2)$ & $0.005(2)$ & $-0.003(2)$ & $0.002(2)$ \\
\hline$C(5)$ & $2 a$ & $0.9158(4)$ & $1.3005(4)$ & $0.3161(2)$ & $0.044(2)$ & $0.047(2)$ & $0.065(2)$ & $0.005(2)$ & $-0.001(2)$ & $-0.006(2)$ \\
\hline$C(6)$ & $2 a$ & $0.8452(3)$ & $1.2291(4)$ & $0.2576(2)$ & $0.043(2)$ & $0.044(2)$ & $0.052(2)$ & $0.011(2)$ & $0.001(2)$ & $0.004(2)$ \\
\hline$C(7)$ & $2 a$ & $0.8132(4)$ & $1.0936(4)$ & $0.2793(2)$ & $0.044(2)$ & $0.049(2)$ & $0.056(2)$ & $0.008(2)$ & $-0.007(2)$ & $-0.001(2)$ \\
\hline$C(8)$ & $2 a$ & $0.9367(3)$ & $1.0254(4)$ & $0.3099(2)$ & $0.043(2)$ & $0.047(2)$ & $0.049(2)$ & $0.010(2)$ & $0.000(2)$ & $0.006(2)$ \\
\hline$C(9)$ & $2 a$ & $0.9051(4)$ & $0.8937(4)$ & $0.3331(2)$ & $0.050(2)$ & $0.050(2)$ & $0.047(2)$ & $0.013(2)$ & $0.002(2)$ & $-0.001(2)$ \\
\hline$C(10)$ & $2 a$ & $0.8084(5)$ & $0.8119(5)$ & $0.3087(2)$ & $0.066(3)$ & $0.055(3)$ & $0.068(3)$ & $0.013(2)$ & $-0.005(2)$ & $-0.001(2)$ \\
\hline$C(11)$ & $2 a$ & $0.7961(5)$ & $0.6891(5)$ & $0.3344(3)$ & $0.075(3)$ & $0.046(3)$ & $0.089(3)$ & $-0.004(2)$ & $0.010(3)$ & -0.009 (3) \\
\hline$C(12)$ & $2 a$ & $0.8808(5)$ & $0.6414(5)$ & $0.3858(3)$ & $0.095(4)$ & $0.046(2)$ & $0.079(3)$ & $0.006(3)$ & $0.021(3)$ & $0.005(3)$ \\
\hline$C(13)$ & $2 a$ & $0.9806(5)$ & $0.7206(5)$ & $0.4099(2)$ & $0.081(3)$ & $0.062(3)$ & $0.057(2)$ & $0.028(3)$ & $0.014(2)$ & $0.018(2)$ \\
\hline$C(14)$ & $2 a$ & $0.9910(4)$ & $0.8465(4)$ & $0.3849(2)$ & $0.052(2)$ & $0.050(2)$ & $0.061(2)$ & $0.013(2)$ & $0.008(2)$ & $0.006(2)$ \\
\hline$C(15)$ & $2 a$ & $1.1126(5)$ & $1.3131(6)$ & $0.3890(3)$ & $0.068(3)$ & $0.067(3)$ & $0.098(4)$ & $-0.015(3)$ & $-0.021(3)$ & $-0.005(3)$ \\
\hline$C(16)$ & $2 a$ & $0.7197(3)$ & $1.2999(4)$ & $0.2355(2)$ & $0.036(2)$ & $0.045(2)$ & $0.058(2)$ & $0.009(2)$ & $0.001(2)$ & $0.010(2)$ \\
\hline$C(17)$ & $2 a$ & $0.6231(3)$ & $1.4592(4)$ & $0.1555(2)$ & $0.040(2)$ & $0.042(2)$ & $0.045(2)$ & $0.012(2)$ & $0.009(1)$ & $0.001(2)$ \\
\hline$C(18)$ & $2 a$ & $0.5029(4)$ & $1.3760(4)$ & $0.1308(2)$ & $0.048(2)$ & $0.038(2)$ & $0.045(2)$ & $0.015(2)$ & $0.009(2)$ & $0.007(2)$ \\
\hline$C(19)$ & $2 a$ & $0.2653(4)$ & $1.3603(4)$ & $0.1610(2)$ & $0.049(2)$ & $0.037(2)$ & $0.074(3)$ & $0.004(2)$ & $0.014(2)$ & $0.009(2)$ \\
\hline$C(20)$ & $2 a$ & $0.1622(4)$ & $1.4594(5)$ & $0.1795(2)$ & $0.044(2)$ & $0.052(3)$ & $0.089(3)$ & $0.007(2)$ & $0.019(2)$ & $0.015(2)$ \\
\hline$C(21)$ & $2 a$ & $0.1130(5)$ & $1.6680(5)$ & $0.2293(3)$ & $0.061(3)$ & $0.064(3)$ & $0.113(4)$ & $0.020(3)$ & $0.039(3)$ & $0.001(3)$ \\
\hline$C(22)$ & $2 a$ & $0.2043(5)$ & $1.7736(6)$ & $0.2534(3)$ & $0.064(3)$ & $0.067(3)$ & $0.095(3)$ & $0.020(3)$ & $0.021(2)$ & $-0.008(3)$ \\
\hline$C(23)$ & $2 a$ & $0.3414(5)$ & $1.7105(5)$ & $0.2673(3)$ & $0.065(3)$ & $0.065(3)$ & $0.074(3)$ & $0.021(2)$ & $0.008(2)$ & $-0.021(3)$ \\
\hline$C(24)$ & $2 a$ & $0.3419(4)$ & $1.6147(4)$ & $0.2105(2)$ & $0.047(2)$ & $0.043(2)$ & $0.058(2)$ & $0.017(2)$ & $0.010(2)$ & $-0.004(2)$ \\
\hline$C(25)$ & $2 a$ & $0.4365(3)$ & $1.5019(4)$ & $0.2201(2)$ & $0.038(2)$ & $0.051(2)$ & $0.042(2)$ & $0.012(2)$ & $0.001(1)$ & $-0.002(2)$ \\
\hline$C(26)$ & $2 a$ & $0.6745(4)$ & $1.5400(4)$ & $0.0983(2)$ & $0.043(2)$ & $0.052(2)$ & $0.054(2)$ & $0.006(2)$ & $0.008(2)$ & $0.005(2)$ \\
\hline$C(27)$ & $2 a$ & $0.5639(5)$ & $1.6078(5)$ & $0.0584(2)$ & $0.063(3)$ & $0.068(3)$ & $0.065(3)$ & $0.013(2)$ & $0.003(2)$ & $0.027(2)$ \\
\hline$C(28)$ & $2 a$ & $0.7780(5)$ & $1.6355(6)$ & $0.1248(3)$ & $0.067(3)$ & $0.075(4)$ & $0.109(4)$ & $-0.015(3)$ & $0.001(3)$ & $0.027(3)$ \\
\hline$C(29)$ & $2 a$ & $0.2471(6)$ & $1.2381(5)$ & $0.2041(3)$ & $0.088(3)$ & $0.043(3)$ & $0.103(4)$ & $0.003(3)$ & $0.040(3)$ & $0.018(3)$ \\
\hline$C(30)$ & $2 a$ & $0.2953(4)$ & $1.1213(4)$ & $0.1717(2)$ & $0.052(2)$ & $0.046(3)$ & $0.079(3)$ & $0.001(2)$ & $0.015(2)$ & $0.009(2)$ \\
\hline$C(31)$ & $2 a$ & $0.2060(9)$ & $1.0730(7)$ & $0.1206(4)$ & $0.135(6)$ & $0.069(4)$ & $0.116(5)$ & $0.010(4)$ & $-0.012(4)$ & $0.003(4)$ \\
\hline$C(32)$ & $2 a$ & $0.234(1)$ & $0.967(1)$ & $0.0811(5)$ & $0.22(1)$ & $0.088(5)$ & $0.130(6)$ & $-0.013(8)$ & $0.019(7)$ & $-0.010(5)$ \\
\hline$C(33)$ & $2 a$ & $0.353(1)$ & $0.913(1)$ & $0.0933(5)$ & $0.153(8)$ & $0.103(7)$ & $0.152(7)$ & $0.056(7)$ & $0.042(6)$ & $0.037(6)$ \\
\hline$C(34)$ & $2 a$ & $0.4476(9)$ & $0.9483(7)$ & $0.1434(7)$ & $0.101(5)$ & $0.058(4)$ & $0.29(1)$ & $-0.002(4)$ & $0.110(7)$ & $0.012(6)$ \\
\hline $\mathrm{C}(35)$ & $2 a$ & $0.4117(7)$ & $1.0626(7)$ & $0.1871(7)$ & $0.068(4)$ & $0.065(4)$ & $0.29(1)$ & $0.001(4)$ & $0.012(5)$ & $0.050(6)$ \\
\hline$O(51)$ & $2 a$ & $0.7784(5)$ & $1.0466(5)$ & $-0.0345(2)$ & $0.120(3)$ & $0.104(3)$ & $0.089(2)$ & $-0.025(3)$ & $-0.001(2)$ & $-0.004(3)$ \\
\hline$C(52)$ & $2 a$ & $0.8802(8)$ & $1.1371(9)$ & $-0.0241(4)$ & $0.118(5)$ & $0.114(6)$ & $0.098(4)$ & $-0.024(5)$ & $0.010(4)$ & $0.012(4)$ \\
\hline$C(53)$ & $2 a$ & $0.864(1)$ & $1.2036(8)$ & $0.0400(4)$ & $0.149(7)$ & $0.082(5)$ & $0.114(5)$ & $-0.017(5)$ & $-0.018(5)$ & $-0.003(5)$ \\
\hline$O(54)$ & $2 a$ & $0.8707(6)$ & $1.1238(7)$ & $0.0938(2)$ & $0.122(4)$ & $0.149(6)$ & $0.092(3)$ & $-0.024(4)$ & $0.000(3)$ & $-0.016(3)$ \\
\hline $\mathrm{C}(55)$ & $2 a$ & $0.7630(8)$ & $1.038(1)$ & $0.0816(4)$ & $0.108(5)$ & $0.172(9)$ & $0.105(5)$ & $-0.030(6)$ & $0.007(4)$ & $0.008(6)$ \\
\hline$C(56)$ & $2 a$ & $0.774(1)$ & $0.966(1)$ & $0.0197(4)$ & $0.172(8)$ & $0.109(6)$ & $0.104(5)$ & $-0.036(7)$ & $-0.003(5)$ & $-0.007(5)$ \\
\hline$O(61)$ & $2 a$ & $0.3037(5)$ & $0.5305(8)$ & $0.4996(3)$ & $0.113(4)$ & $0.191(7)$ & $0.148(5)$ & $0.060(5)$ & $0.022(3)$ & $0.087(5)$ \\
\hline$C(62)$ & $2 a$ & $0.397(1)$ & $0.556(2)$ & $0.4534(5)$ & $0.19(1)$ & $0.28(2)$ & $0.116(6)$ & $0.09(1)$ & $0.048(7)$ & $0.11(1)$ \\
\hline$C(63)$ & $2 a$ & $0.525(1)$ & $0.574(3)$ & $0.480(2)$ & $0.12(1)$ & $0.37(4)$ & $0.64(5)$ & $0.05(2)$ & $0.14(2)$ & $0.32(4)$ \\
\hline$O(64)$ & $2 a$ & $0.5615(8)$ & $0.468(2)$ & $0.5189(7)$ & $0.102(5)$ & $0.36(2)$ & $0.32(1)$ & $0.074(9)$ & $0.003(6)$ & $0.05(2)$ \\
\hline$C(65)$ & $2 a$ & $0.474(1)$ & $0.442(2)$ & $0.5690(5)$ & $0.18(1)$ & $0.23(2)$ & $0.130(7)$ & $0.08(1)$ & $-0.042(7)$ & $0.038(9)$ \\
\hline$C(66)$ & $2 a$ & $0.346(1)$ & $0.437(2)$ & $0.5413(6)$ & $0.139(8)$ & $0.24(2)$ & $0.169(9)$ & $0.05(1)$ & $0.053(7)$ & $0.11(1)$ \\
\hline
\end{tabular}

